\title{
Two-Dimensional Exciton Diffusion in an HJ-Aggregate of Naphthobisoxadiazole-Based Copolymer Films
}

\section{AUTHOR(S):}

Murata, Yasuhiro; Takeyama, Taiki; Sakamoto, Yuji; Yamaguchi, Kento; Tamai, Yasunari; Ohkita, Hideo

\section{CITATION:}

Murata, Yasuhiro ...[et al]. Two-Dimensional Exciton Diffusion in an HJ-Aggregate of Naphthobisoxadiazole-Based Copolymer Films. The Journal of Physical Chemistry C 2020, 124(24): 13063-13070

\section{ISSUE DATE:}

2020-06-18

\section{URL:}

http://hdl.handle.net/2433/254218

\section{RIGHT:}

This document is the Accepted Manuscript version of a Published Work that appeared in final form in The Journal of Physical Chemistry C, copyright (C) American Chemical Society after peer review and technical editing by the publisher To access the final edited and published work see https://doi.org/10.1021/acs.jpcc.0c04057.; The full-text file will be made open to the public on 1 June 2021 in accordance with publisher's 'Terms and Conditions for Self-Archiving'.; $こ \sigma$ 論文は出版社版でありません。引用の際には出版社版をご確認ご利用ください。; This is not the published version. Please cite only the published version. 


\section{Two-Dimensional Exciton Diffusion in an HJ-}
Aggregate
of
Naphthobisoxadiazole-based

\section{Copolymer Films}

AUTHOR NAMES;

Yasuhiro Murata,${ }^{\dagger}$ Taiki Takeyama,${ }^{\dagger}$ Yuji Sakamoto,${ }^{\dagger}$ Kento Yamaguchi,${ }^{\dagger}$ Yasunari Tamai $,{ }^{,}, \dagger$ and Hideo Ohkita

AUTHOR ADDRESS;

'Department of Polymer Chemistry, Graduate School of Engineering, Kyoto University, Katsura, Nishikyo, Kyoto 615-8510, Japan

\#apan Science and Technology Agency (JST), PRESTO, 4-1-8 Honcho Kawaguchi, Saitama 3320012, Japan 


\section{ABSTRACT}

Understanding exciton diffusion properties is essential for organic optoelectronics such as organic solar cells (OSCs) because excitons in semiconducting polymer solids need to migrate as quasiparticles to a donor/acceptor interface to dissociate into charges in OSCs. Previous studies mainly focused on the diffusion length of excitons. In addition, the dimensionality of exciton diffusion is an important property because OSCs usually consist of semi-crystalline conjugated polymers wherein anisotropic exciton diffusion is expected. Herein we investigate the dimensionality of exciton diffusion in a naphthobisoxadiazole-based low-bandgap polymer PNOz4T through transient absorption spectroscopy. We track the time evolution of the excitation intensity dependent transient signals caused by singlet-singlet exciton annihilation. By analyzing the time dependence of the annihilation rate coefficient, we find that excitons generated in PNOz4T crystalline domains show anisotropic two-dimensional diffusion along the backbone and $\pi$-stacking directions, which can be rationalized by comparable intra- and interchain exciton couplings. 


\section{TOC GRAPHICS}

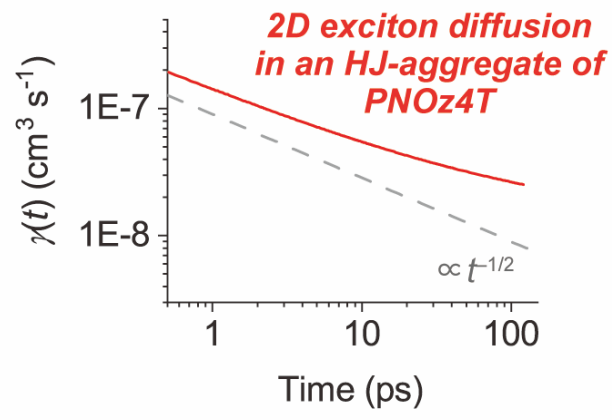

KEYWORDS: energy transfer, exciton migration, organic solar cells, polymer aggregates, singlet-singlet exciton annihilation, transient absorption spectroscopy 


\section{Introduction}

Over the past three decades, conjugated polymers have attracted considerable attention as core materials for organic optoelectronics such as organic solar cells (OSCs) $\cdot{ }^{1-4}$ When we shine a light on semiconducting conjugated polymers, singlet excitons (i.e., Coulombically bound electronhole pairs) are formed instead of free charge carriers due to their low dielectric constants. Hence, these polymers cannot generate a photocurrent by themselves, which is in sharp contrast to inorganic semiconductors. Excitons need to diffuse to a donor/acceptor (DA) interface where they separate into free charge carriers. Therefore, exciton diffusion properties are essential for solar cell

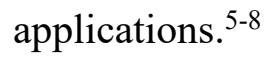

Many researchers including us have reported exciton diffusion lengths of conjugated polymers ${ }^{9-}$ ${ }^{16}$ and pointed out the importance of film morphology. ${ }^{16-22}$ For example, we reported that the exciton diffusion length of poly(3-hexylthiophene) (P3HT) films strongly depend on their crystallinity. ${ }^{21,22} \mathrm{We}$ observed that the diffusion length of P3HT increased up to four times with increasing crystallinity $(4.8 \mathrm{~nm}$ in fully amorphous regiorandom P3HT film to $20 \mathrm{~nm}$ in highly crystalline regioregular $\mathrm{P} 3 \mathrm{HT}$ film). In contrast, the dimensionality of exciton diffusion in crystalline conjugated polymer films has not be extensively studied, whereas it has been well established for organic small molecules. ${ }^{23-26}$ Although we can safely consider that excitons diffuse randomly in all directions in fully amorphous isotropic materials, this would not be the case for semi-crystalline polymer films because the crystalline structure of these polymers is highly anisotropic, where diffusion coefficients along each direction are supposed to be different. One example reported by us shows that singlet excitons generated in P3HT crystalline phases predominantly diffuse along the $\pi$-stacking direction, which is rationalized by a strong interchain exciton coupling in $\mathrm{P} 3 \mathrm{HT} .{ }^{6,21}$ There are some other reports that suggest efficient interchain exciton 
hopping as well. ${ }^{27-29}$ On the other hand, the determinant factors and control mechanism of the dimensionality of exciton diffusion in conjugated polymer solids are not completely elucidated. It is important to understand what parameters determine the dimensionality of exciton diffusion in crystalline polymer films.

Herein, we study the exciton diffusion dynamics in a novel low-bandgap polymer PNOz4T (chemical structure is shown in Figure 1a). PNOz4T is a D-A copolymer of quaterthiophene (4T) and naphthobisoxadiazole (NOz) with a deep highest occupied molecular orbital (HOMO) energy of $-5.48 \mathrm{eV},{ }^{30}$ achieving a high open-circuit voltage $\left(V_{\mathrm{OC}}\right)$ of $\sim 1 \mathrm{~V}$ with efficient photocurrent generation efficiency in OSCs combined with fullerene derivatives $\left(\mathrm{PC}_{61} \mathrm{BM}\right.$ and $\left.\mathrm{PC}_{71} \mathrm{BM}\right){ }^{31}$ PNOz4T forms $\pi$-stacked crystalline structures similar to P3HT as reported previously. ${ }^{30}$ On the other hand, unlike P3HT that exhibits typical H-aggregate behavior, ${ }^{32-34}$ PNOz4T displays the HJaggregate behavior as will be shown later. We performed transient absorption (TA) measurements to track the time evolution of the exciton density. We found clear signals of anisotropic twodimensional (2D) exciton diffusion in PNOz4T crystalline domains.

\section{Experimental Methods}

\section{Sample Preparation}

Thin films were prepared onto quartz substrates by spin-coating from a chlorobenzene (CB) solution. The film thickness was approximately $70 \mathrm{~nm}$. For TA measurements, the sample films were encapsulated in a $\mathrm{N}_{2}$-purged glove box.

\section{Measurements}


UV-visible absorption and photoluminescence spectra were measured with a UV-visible spectrophotometer (Hitachi, U-4100) and a fluorescence spectrophotometer (Horiba Jobin Yvon, NanoLog) equipped with a photomultiplier tube (Hamamatsu, R928P) and a liquid-nitrogencooled InGaAs near-IR array detector (Horiba Jobin Yvon, Symphony II). The excitation wavelength was set at $700 \mathrm{~nm}$.

TA measurements were performed with a pump and probe transient spectroscopy system. This system consists of a regenerative amplified Ti:sapphire laser (Spectra-Physics, Hurricane) and a TA spectrometer (Ultrafast systems, Helios). The amplified Ti:sapphire laser provided $800 \mathrm{~nm}$ fundamental pulses at a repetition rate of $1 \mathrm{kHz}$ with the pulse width of $100 \mathrm{fs}$ (fwhm), which were split into two optical beams with a beam splitter to generate pump and probe pulses. One fundamental beam was converted into white light employed as probe pulses in the wavelength region from 800 to $1600 \mathrm{~nm}$. The other fundamental beam was used as pump pulses at $750 \mathrm{~nm}$ after conversion with an ultrafast optical parametric amplifier (Spectra-Physics, TOPAS). The pump pulses were modulated mechanically at the repetition rate of $500 \mathrm{~Hz}$. Temporal evolution of the probe intensity was recorded with an InGaAs linear diode array sensor (Ultrafast Systems, SPEC-NIR). TA spectra and decays were collected over the time range of $-5 \mathrm{ps}$ to $3 \mathrm{~ns}$. Typically, 2500 laser shots were averaged at each delay time to obtain a detectable absorbance change as small as $\sim 10^{-4}$. In order to cancel out orientation effects on the dynamics, the polarization direction of the linearly polarized probe pulse was set at the magic angle of $54.7^{\circ}$ with respect to that of the pump pulse.

Temperature dependence measurements were conducted with a liquid-nitrogen-cooled cryostat (Oxford Instruments, Optistat DN-V) for steady-state absorption and TA measurements and a Cryofree ${ }^{\circledR}$ cryostat (Oxford Instruments, Optistat Dry BL4) for PL measurements. 


\section{Analytical Method}

In order to extract the time dependent annihilation rate coefficients $\gamma(t)$, we used a numerical method based on a genetic algorithm (GA). In this algorithm, a population of time traces $\gamma_{0}^{\prime}(t)$ is generated randomly. Subsequently, those candidates are iteratively evolved towards better solutions $\gamma^{\prime}{ }_{i}(t)$. During each successive generation, the existing population is bred to form a next generation using a survival of the fittest approach. The fitness $f$ is calculated as the inverse of the sum of the squared residual as $f=1 /\left(n(t)-n^{\prime}{ }_{i}(t)\right)^{2}$, where $n(t)$ is a measured exciton decay kinetics and $n^{\prime}{ }_{i}(t)$ is obtained by Equation 3 in the next section using $\gamma^{\prime}{ }_{i}(t)$. For each new solution to be produced, a pair of "parent" is randomly selected for breeding, and a "child" is produced by crossover and mutation.

\section{Results and Discussion}

\section{HJ-aggregate behavior of $\mathrm{PNO}_{4} 4 \mathrm{~T}$}

The crystalline structure of PNOz4T, which was previously revealed by grazing incidence wideangle X-ray diffraction (GIXD) measurements, ${ }^{30}$ is similar to that of P3HT. ${ }^{35,36}$ The $\pi$ - $\pi$ stacking distance of PNOz4T crystals is $3.5 \AA$, which is slightly smaller than that of P3HT (3.8 $\AA$ ). The lamellar distance of $22 \AA$ in PNOz4T is larger than that of P3HT (16 $⿱$ P) because of comparatively long side chains. It is well known that P3HT exhibits the H-aggregate behavior due to strong interchain exciton coupling where the 0-0 emission band decreases monotonically with decreasing temperature. ${ }^{37,38}$ However, the photophysical properties of PNOz4T are completely different from that of P3HT. Figures $1 \mathrm{~b}$ and $1 \mathrm{c}$ show the temperature dependence of the photoluminescence (PL) spectra of a PNOz4T thin film. As shown in the figure, the PL spectra of the PNOz4T film shows 
a

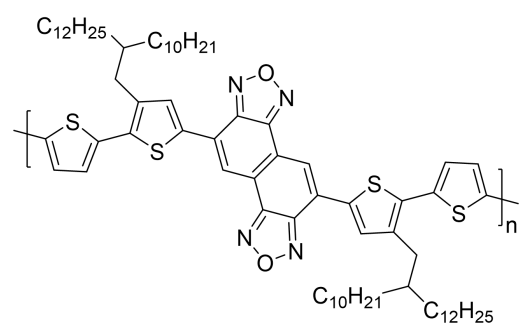

b
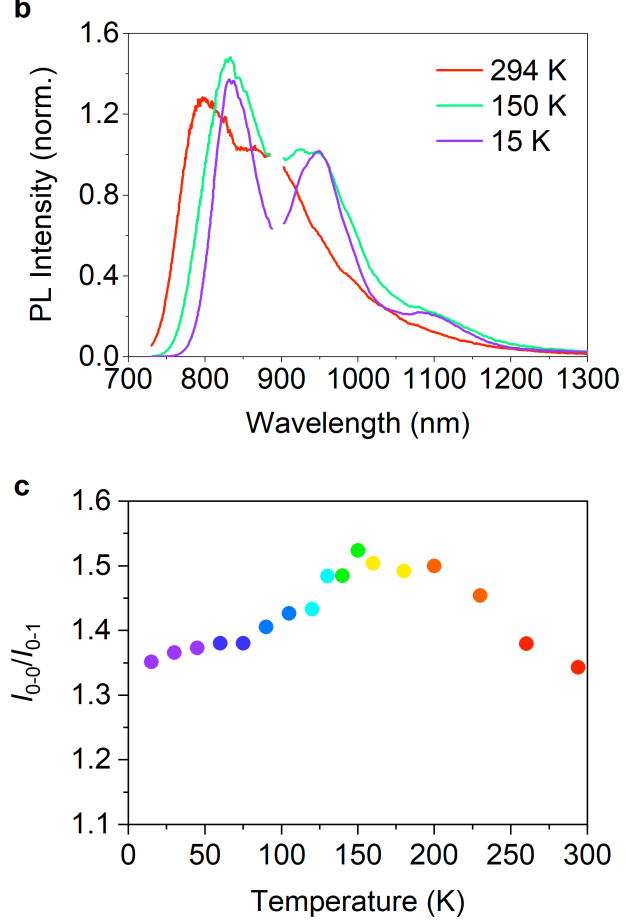

Figure 1. (a) Chemical structure of PNOz4T. (b) Normalized PL spectra at various temperatures. PL data at around $900 \mathrm{~nm}$ were masked due to an artifact signal from the equipment (unmasked data are shown in the Supporting Information). (c) Temperature dependence of the PL intensity ratio $I_{0-0} / I_{0-1}$.

characteristic vibronic bands ranging from 800 to $900 \mathrm{~nm}$. As shown in panel b, the PL spectra shift toward red with decreasing temperature, suggesting that conformational disorder would be reduced at lower temperatures. On the other hand, the highest energy band at approximately 800 
$\mathrm{nm}$ increased relative to the lower-energy sidebands with decreasing temperature until $150 \mathrm{~K}$, and then decreased with decreasing temperature under $150 \mathrm{~K}$. This complex behavior cannot be rationalized solely by the reduction of conformational disorder. Instead, the complex temperature dependence has been theoretically predicted in the HJ-aggregate model proposed by Spano and his co-workers, ${ }^{39,40}$ wherein they considered intrachain interaction as J-coupling, while interchain interaction was described as H-coupling. ${ }^{39,40}$ In the HJ-aggregate model, the PL intensity ratio $I_{0 \text { - }}$ ${ }_{0} / I_{0-1}$ peaks at a threshold temperature because the temperature dependence of $I_{0-0} / I_{0-1}$ consists of an H-like thermally activated term and a J-like thermally deactivated term. Although P3HT pristine films show the H-aggregate behavior, highly ordered P3HT nanofibers exhibit the HJ-aggregate properties, as reported previously. ${ }^{39-41}$ Thus, we conclude that PNOz4T is likely to form HJaggregates owing to the higher backbone planarity compared to P3HT. The ratio between intraand interchain exciton couplings $\left(J_{\text {intra/inter }}\right)$ was estimated by Equation 1.

$$
\left(\frac{I_{0-0}}{I_{0-1}}\right)_{\max }=\frac{1.35}{\lambda_{0}^{2}} \sqrt{\frac{\left|J_{\text {intra }}\right|}{J_{\text {inter }}}}
$$

where $\lambda_{0}$ is a Huang-Rhys factor and assumed to be equal to that of P3HT $\left(\lambda_{0}^{2}=1.5\right)^{39}$ because PNOz4T mainly consists of thiophene units. From the $I_{0-0} / I_{0-1}$ at the peak temperature of $150 \mathrm{~K}$, the ratio $\left|J_{\text {intra }}\right| / J_{\text {inter }}$ was estimated to be $\sim 2.8$, suggesting that the intrachain exciton coupling is slightly larger than the interchain exciton coupling in PNOz4T as opposed to P3HT.

\section{Analytical model}

Various experimental methods, such as surface fluorescence quenching using bilayer films, ${ }^{9,10,16}$ bulk fluorescence quenching using blend films of polymers and small amounts of quenchers, ${ }^{11,12,14}$ 
and singlet-singlet exciton annihilation $(\mathrm{SSA})^{16,24,42}$, have been used to measure exciton diffusion properties. These techniques have different advantages and disadvantages. Previous studies have revealed that the surface/bulk quenching methods underestimate the diffusion length for semicrystalline polymer films compared to that with SSA, whereas all methods give consistent results for amorphous films. ${ }^{21,43}$ This is because the spatial and temporal distribution of exciton diffusion dynamics is averaged in the quenching methods, which is likely not the case for semi-crystalline polymer films as mentioned above. We have shown that SSA with the selective excitation of relatively low energy sites is essential to reveal exciton diffusion dynamics in semi-crystalline polymer films. ${ }^{21}$ As we try to understand the dimensionality of exciton diffusion in an HJaggregate of PNOz4T, the SSA method is the most suitable for this study. Figure 2a shows TA spectra of the PNOz4T film. The excitation wavelength was set at $750 \mathrm{~nm}$ to selectively excite relatively low energy sites of PNOz4T crystalline phases. A broad photoinduced absorption band in the near-IR region observed immediately after photoexcitation is attributable to singlet excitons, as reported previously. ${ }^{31}$ Other transient species such as polarons and triplet excitons were not remarkable at least under the experimental condition employed in this study. Figure $2 \mathrm{~b}$ shows the excitation intensity dependence of exciton decay kinetics measured at $1400 \mathrm{~nm}$. Singlet excitons decayed faster with increasing excitation intensity, suggesting the presence of an additional deactivation pathway through SSA. On the other hand, the exciton decay was independent of the excitation intensity at a later stage (>150 ps), where excitons decayed monomolecularly with a time constant of $\sim 230$ ps at $294 \mathrm{~K}$.

The rate equation for singlet excitons including SSA is given as

$$
\frac{\mathrm{d} n(t)}{\mathrm{d} t}=-k n(t)-f \gamma(t) n^{2}(t)
$$


a
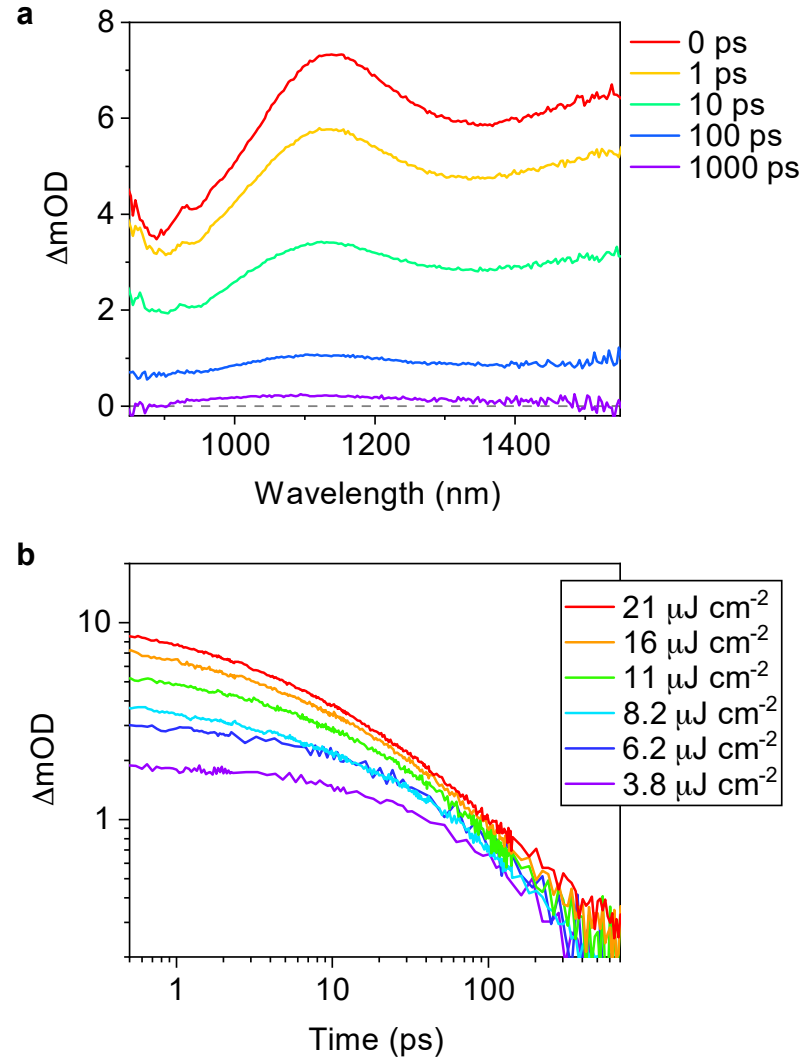

Figure 2. (a) TA spectra of the PNOz4T film excited at a wavelength of $750 \mathrm{~nm}$ with a fluence of $11 \mu \mathrm{J} \mathrm{cm}^{-2}$. (b) Excitation intensity dependence of singlet exciton decay kinetics monitored at $1400 \mathrm{~nm}$.

where $n(t), k$, and $\gamma(t)$ are the exciton density at time $t$ after photoexcitation, the rate constant of monomolecular deactivation given by inverse of the exciton lifetime, and the rate coefficient of SSA, respectively. Here, we assumed time-independent intrinsic decay rate constant $k$. This assumption is sometimes not valid for conjugated polymer films, wherein multi-exponential behavior is observed. However, this assumption does not affect our conclusion showing later because SSA is dominant rather than intrinsic decay at early times, meaning that a small variation in $k$ because of disorder does not make substantial contribution to the overall decay kinetics. We 
also assumed prefactor $f$ to be $1 / 2$, meaning that only one of the two excitons decays by SSA ( $\mathrm{S}_{1}$ $+\mathrm{S}_{1} \rightarrow \mathrm{S}_{\mathrm{n}}+\mathrm{S}_{0} \rightarrow \mathrm{S}_{1}+\mathrm{S}_{0}+$ phonons). This equation is analytically solved as

$$
n(t)=\frac{n_{0} \exp (-k t)}{1+\frac{n_{0}}{2} \int_{0}^{t} \gamma(t) \exp (-k t) \mathrm{d} t}
$$

where $n_{0}$ represents the exciton density at time zero (the detailed method to evaluate $n_{0}$ is described in the Supporting Information).

Because SSA is a diffusion-limited process, the rate coefficient $\gamma(t)$ is strongly dependent on the diffusion properties of excitons. As summarized previously, the rate coefficient $\gamma(t)$ is a function of the dimensionality of exciton diffusion. ${ }^{6,21}$ For example, the $\gamma(t)$ in the isotropic $3 \mathrm{D}$ diffusion system, wherein excitons diffuse in all directions with an isotropic diffusion constant $D$, is given by

$$
\gamma_{\mathrm{iso3D}}(t)=8 \pi D R\left(1+\frac{R}{\sqrt{2 \pi D t}}\right)
$$

where $R$ is effective reaction radius of excitons. When $t>R^{2} /(2 \pi D)$, the $\gamma_{\text {iso3D }}(t)$ can be expressed as the time-independent formula $\gamma_{\text {iso3D }}=8 \pi D R$.

The $\gamma(t)$ for the 1D system is also simply given as

$$
\gamma_{1 \mathrm{D}}(t)=4 \pi D R \frac{R}{\sqrt{2 \pi D t}}
$$

Therefore, the $\gamma(t)$ shows the $t^{-1 / 2}$ dependence over the entire time domain in the $1 \mathrm{D}$ system.

In contrast, we are not able to obtain a simple formula for the $2 \mathrm{D}$ system even when diffusion constants along the two directions are the same because of symmetry reduction (see the Supporting 
Information). The situation is more complicated if we consider anisotropic 2D diffusion, wherein diffusion constants along the two directions are different. Therefore, detailed discussion to express analytical formula for the $2 \mathrm{D}$ system is beyond the scope of this study. However, it is reasonable to think that the $\gamma(t)$ shows $t^{-\alpha}(0<\alpha<1 / 2)$ dependence in the anisotropic system because two extreme cases (isotropic 3D and $1 \mathrm{D}$ systems) show $t^{0}$ and $t^{-1 / 2}$ dependences, respectively. In summary, it shows the $t^{-1 / 2}$ dependence over the entire time domain in the $1 \mathrm{D}$ system, $t^{-\alpha}(0<\alpha<$ $1 / 2$ ) dependence in the 2D system, and becomes time-independent in the isotropic $3 \mathrm{D}$ system. In other words, $\alpha$ becomes larger as the anisotropy of the exciton diffusion becomes apparent. This indicates that the time dependence of $\gamma(t)$ is a direct measure of the dimensionality of the exciton diffusion. $^{21}$

Many previous studies including ours performed a forward analysis using normal least-squares methods with rate equations such as Equation 3 with Equation 4 or 5 to obtain diffusion parameters. On the other hand, we used a genetic algorithm because the least-squares methods do not work well in this study. This is because rate equations for the $2 \mathrm{D}$ system is fairly complicated than those of the $1 \mathrm{D}$ and $3 \mathrm{D}$ systems as mentioned above. In that situation, the forward analysis usually fails to provide a global minimum solution. Rather, inverse analyses are more effective, as will be shown later. Therefore, we performed an inverse analysis using a genetic algorithm with Equation 3 to extract a time dependence of $\gamma(t) .{ }^{44-47}$

\section{Two-dimensional exciton diffusion}


The algorithm we used iteratively finds optimized $\gamma(t)$ that gives the best fitted $n(t)$ (the details of this algorithm are described in the Experimental Methods section ). The red line in Figure 3a represents a reproduced $n(t)$ curve calculated with the best $\gamma(t)$. The reproduced $n(t)$ is well-fitted with the decay kinetics measured (black line). Note that we analyzed only $\leq 150$ ps time range
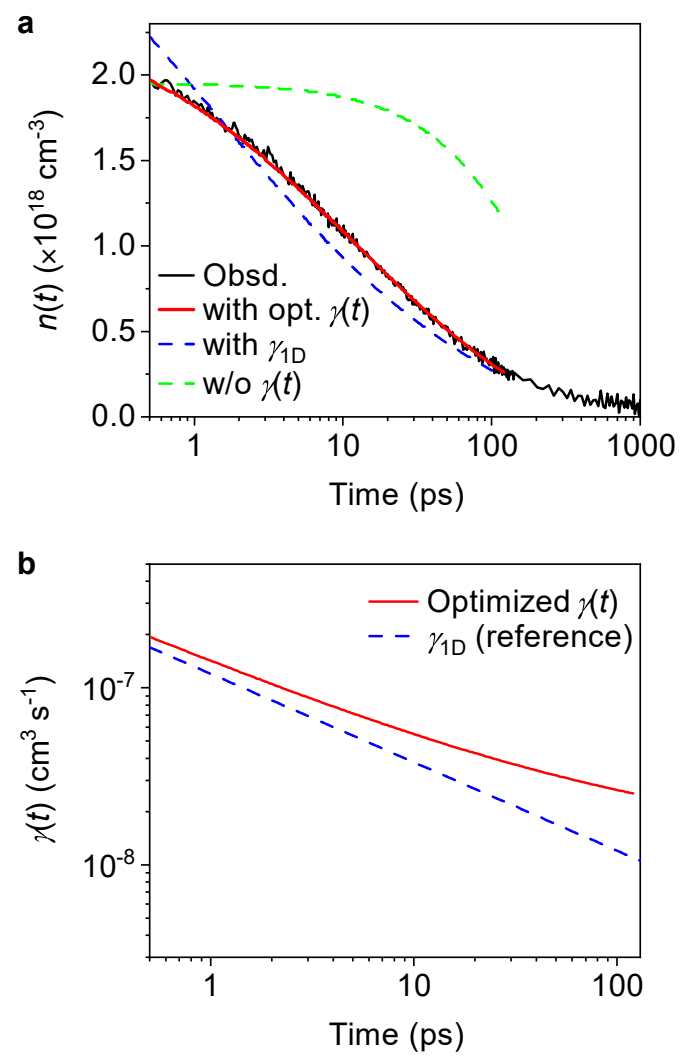

Figure 3. (a) Measured (black) and reproduced (red) decay kinetics using optimized annihilation rate coefficient $\gamma(t)$ (panel b, red line) for singlet excitons monitored at $1400 \mathrm{~nm}$. Broken lines exemplify how a change in the behavior of $\gamma(t)$ would change the fit: (blue) calculated with the $t^{-1 / 2}$ dependence, and (green) calculated without $\gamma(t)$ (i.e., in the absence of SSA). (b) Optimized annihilation rate coefficient $\gamma(t)$. The broken line represents the $t^{-1 / 2}$ dependence as a guide for the eye. Calculations were conducted only $\leq 150$ ps time range as mentioned in the main text. 
because the exciton decay kinetics was independent of the excitation intensity after $150 \mathrm{ps}$, as shown in Figure 2b. In other words, intrinsic decay is dominant rather than SSA after 150 ps, indicating that it is meaningless to discuss the extracted $\gamma(t)$ after 150 ps because the $\gamma(t)$ has no impact on the decay kinetics (i.e., the second term in the denominator of Equation 3 will be fairly smaller than 1 after $150 \mathrm{ps})$. Figure $3 \mathrm{~b}$ shows $\log -\log$ plots of iteratively optimized $\gamma(t)$ against time $t$. The slope is gentler than $-1 / 2$, which is different from that of $\mathrm{P} 3 \mathrm{HT}$ in which the slope is $-1 / 2$ over the entire time domain (i.e., $1 \mathrm{D}$ diffusion). ${ }^{21}$ This is supported by the blue broken line in panel a. Broken lines in panel a exemplify how a change in the behavior of $\gamma(t)$ would change the fit. As clearly shown in the figure, neither the blue line, calculated with the $t^{-1 / 2}$ dependence, nor the green line, calculated without $\gamma(t)$ (i.e., in the absence of SSA), provide better results than the red line. These results strongly indicate that the dimensionality of the exciton diffusion in the PNOz4T crystalline phase is different from that of P3HT, i.e., singlet excitons would diffuse 2- or 3-dimensionally in the PNOz4T crystalline phase. Because of the long lamellar distance, ${ }^{18}$ we safely rule out exciton diffusions along the lamellar direction. Therefore, we conclude that the singlet excitons in PNOz4T crystalline phases diffuse two-dimensionally along the backbone and $\pi$-stacking directions. This would be rationalized by comparable intra- and interchain exciton couplings in PNOz4T crystalline phases as mentioned above.

We also measured the temperature dependence of exciton diffusion dynamics. Figure 4 shows the temperature dependence of the decay kinetics and extracted annihilation rate coefficient $\gamma(t)$ ( $n(t)$ and $\gamma(t)$ at other temperatures are shown in the Supporting Information). Interestingly, as shown in column $\mathrm{b}$, the $\alpha$ gradually increases (i.e., the slope $-\alpha$ gradually decreases) with decreasing temperature, and finally reaches $-1 / 2$ under $120 \mathrm{~K}$, indicating that $1 \mathrm{D}$ diffusion is dominant at low temperatures. These findings suggest that although excitons in PNOz4T can 
a

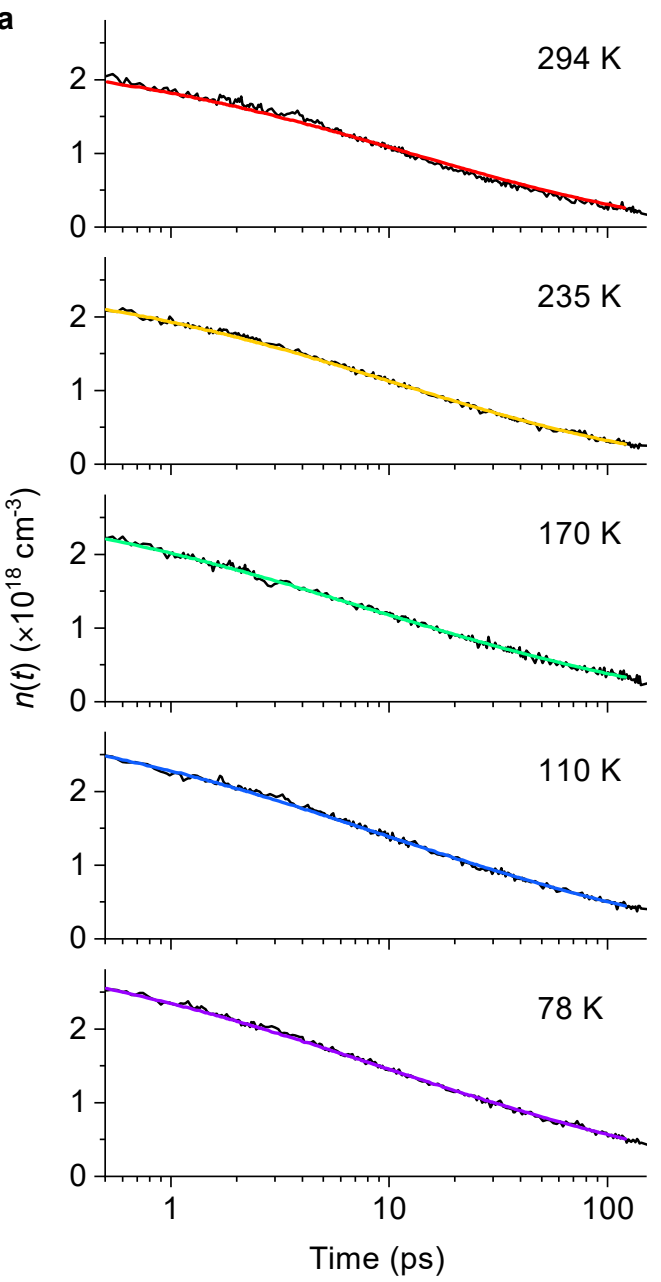

b
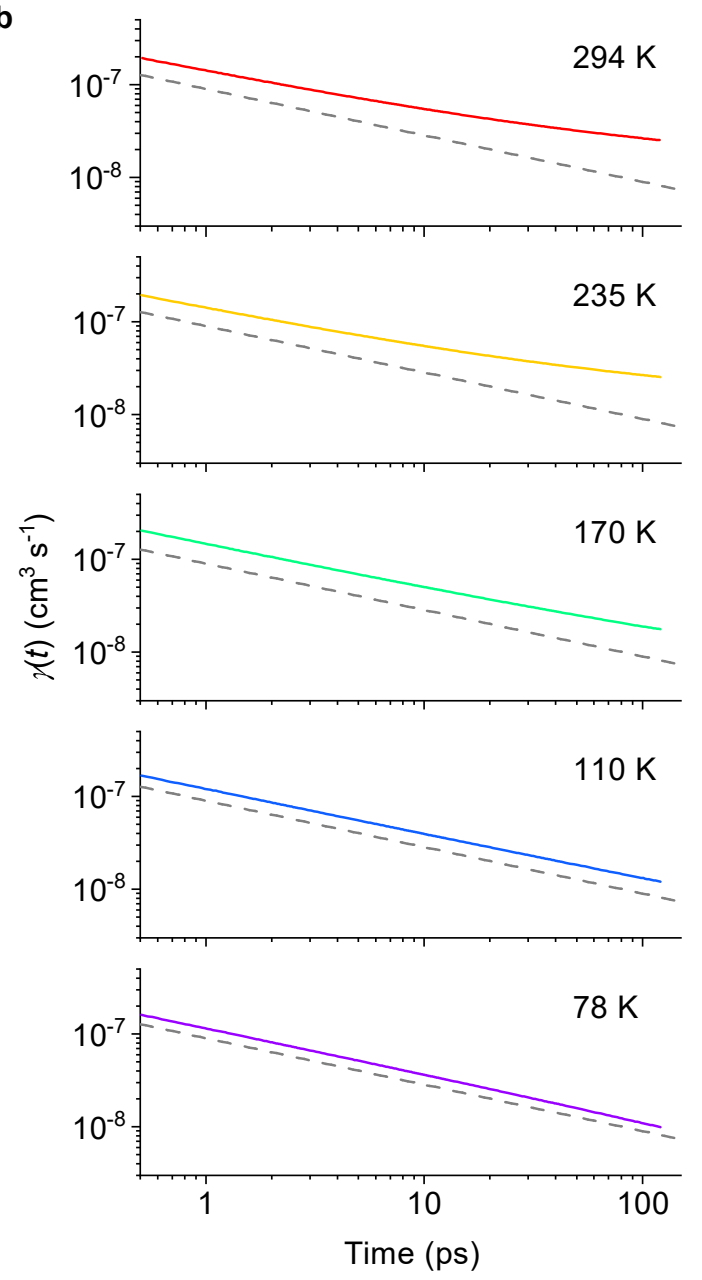

Figure 4. (a) Measured (black lines) and reproduced (thick colored lines) decay kinetics using optimized annihilation rate coefficient $\gamma(t)$ (column b) for singlet excitons monitored at 1400 nm. Temperature was varied as indicated. (b) Optimized annihilation rate coefficient $\gamma(t)$ at each temperature. The broken line in each panel represents the $t^{-1 / 2}$ dependence as a guide for the eye.

diffuse in both intra- and interchain directions at room temperature $(294 \mathrm{~K})$, the activation energies for those directions should be different. At low temperatures, the exciton diffusion along one 
direction is much slower than that in the other direction; hence, it can be disregarded.

\section{Exciton dynamics in nanoaggregates}

To reveal the direction that has the lower activation energy, we performed TA measurements for PNOz4T in a chlorobenzene (CB) solution. As shown in Figure 5a, the absorption spectrum of PNOz4T in the CB solution is completely different from that in a chloronaphthalene $(\mathrm{CN})$ solution. Rather, it is consistent with that of the film state. This suggests that PNOz4T does not fully dissolve in the $\mathrm{CB}$ solution because of poor solubility, but forms nanoaggregates in the $\mathrm{CB}$ solution, as reported previously. ${ }^{30}$ This is supported by TA measurements as shown in panel b. Excitation intensity independent decay was observed for singlet excitons in the $\mathrm{CN}$ solution. In contrast, excitons in the $\mathrm{CB}$ solution decayed faster than that in the $\mathrm{CN}$ solution and became faster with increasing the excitation intensity, which is a sign of SSA in nanoaggregates because SSA hardly occurs in dilute solutions of isolated polymers like the $\mathrm{CN}$ solution. Figure $5 \mathrm{c}$ shows optimized $\gamma(t)$ for the CB solution. Interestingly, the time dependence of $\gamma(t)$ was clearly different from that of the film state even though there was no large difference in the absorption and PL spectra between them: $\gamma(t)$ of the CB solution follows the $t^{-1 / 2}$ dependence, suggesting 1D exciton diffusion. This result indicates that excitons in the nanoaggregates are able to diffuse in only one of the two directions due to spatial constraint. Because the relative strength of each vibronic band in the absorption and PL spectra (see the Supporting Information) of the CB solution is consistent with that of the film state, the backbone planarity and effective conjugation length of PNOz4T in the nanoaggregates are supposed to be comparable to that of the film state. In other words, excitons would be able to diffuse along the backbone direction in the $\mathrm{CB}$ solution as is the case with the film state. Therefore, we consider that excitons in the nanoaggregates diffuse predominantly along 
the backbone direction and cannot diffuse along the $\pi$-stacking direction because of the spatial confinement. Figure $5 \mathrm{~d}$ shows the temperature dependence of the exciton decay kinetics of the PNOz4T nanoaggregates. Interestingly, negligible temperature dependence was observed for the PNOz4T nanoaggregates as opposed to the film state, suggesting that the activation energy of the exciton diffusion along the backbone direction is considerably low. This result suggests that, at

a

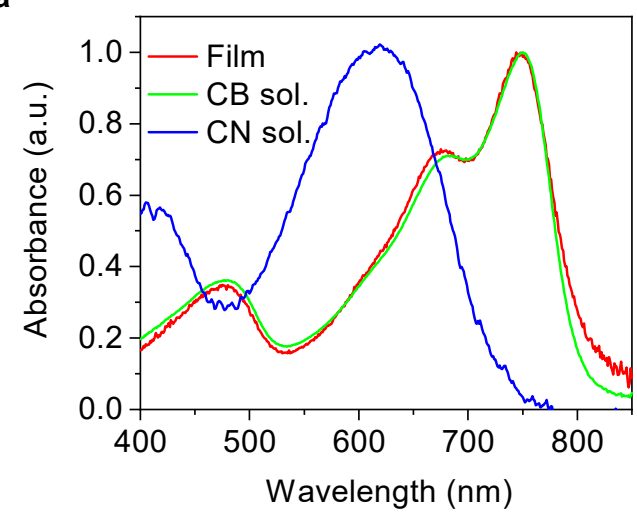

C

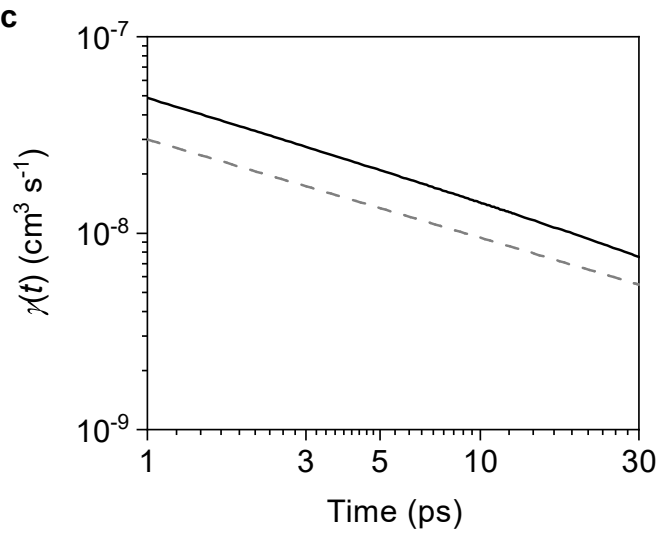

b

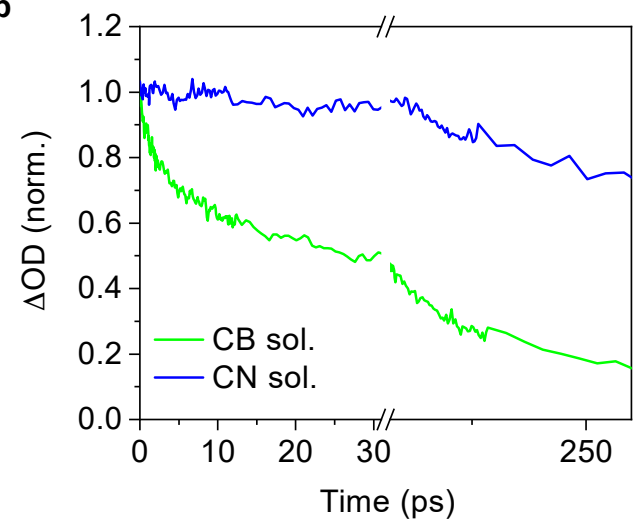

d

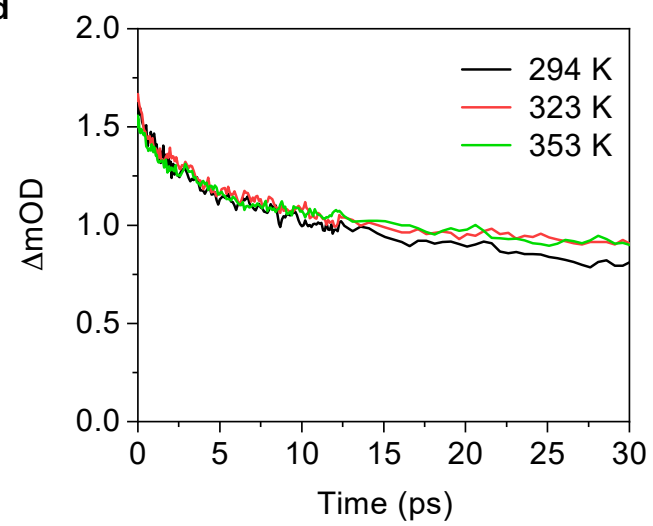

Figure 5. (a) Absorption spectra of PNOz4T in the CB solution (green), and in the CN solution (blue). The red line represents the absorption spectrum of the PNOz4T film. (b) Exciton decay kinetics in the $\mathrm{CB}$ (green) and $\mathrm{CN}$ (blue) solutions. (c) Optimized annihilation rate coefficient $\gamma(t)$ for the CB solution. The broken line represents the $t^{-1 / 2}$ dependence as a guide for the eye. (d) Temperature dependence of the exciton decay kinetics in the CB solution. 
low temperatures, excitons in PNOz4T films predominantly diffuse along the backbone direction with considerably small activation energy. Exciton diffusion along the $\pi$-stacking direction becomes important only at high temperatures because of the large activation energy in PNOz4T films.

\section{Conclusion}

We have studied the diffusion dynamics of singlet excitons in a novel low-bandgap donor polymer PNOz4T in film state. Applying numerical calculations to excitation intensity dependent exciton decay kinetics, we found that singlet excitons generated in PNOz4T crystalline domains diffuse along both the backbone and $\pi$-stacking directions. This is contrary to the $1 \mathrm{D}$ diffusion in P3HT crystalline domains. What controls the dimensionality of exciton diffusion can be rationalized by the ratio between intra- and interchain exciton couplings. As PNOz4T shows comparable intra- and interchain exciton couplings, excitons in PNOz4T are able to diffuse in both intra- and interchain directions, whereas strong interchain exciton coupling in P3HT lets excitons predominantly diffuse along the $\pi$-stacking direction. To the best of our knowledge, this study provides for the first time a clear experimental evidence of $2 \mathrm{D}$ exciton diffusion along the backbone and $\pi$-stacking directions in semi-crystalline conjugated polymer films. Our findings suggest that we would be able to control the direction of the exciton motion by modifying backbone planarity of conjugated chains. 


\section{Supporting Information}

Supporting Information is available free of charge via the Internet at http://pubs.acs.org.

Temperature dependence of absorption and PL spectra, Details of TA measurements, and PL spectra of PNOz4T in CB solution.

\section{AUTHOR INFORMATION}

\section{Corresponding Author}

*YT: E-mail: tamai@photo.polym.kyoto-u.ac.jp

\section{Author Contributions}

The manuscript was written through contributions of all authors. All authors have given approval to the final version of the manuscript.

\section{Notes}

The authors declare no competing financial interests.

\section{ORCID}

Yasunari Tamai: 0000-0002-3074-0208

Hideo Ohkita: 0000-0002-7403-3492 


\section{ACKNOWLEDGMENTS}

We are grateful to Prof Itaru Osaka at Hiroshima University for providing us with PNOz4T. This study was partly supported by JST PRESTO Grant Number JPMJPR1874, JST ALCA Grant Number JPMJAL1404, JSPS KAKENHI Grant Number JP26248033 and 17K14527, and PII Foundation of Institute for Chemical Fibers, Japan. 


\section{References}

1. Clarke, T. M.; Durrant, J. R. Charge Photogeneration in Organic Solar Cells. Chem. Rev. 2010, $110,6736-6767$.

2. Lu, L.; Zheng, T.; Wu, Q.; Schneider, A. M.; Zhao, D.; Yu, L. Recent Advances in Bulk Heterojunction Polymer Solar Cells. Chem. Rev. 2015, 115, 12666-12731.

3. Hou, J.; Inganäs, O.; Friend, R. H.; Gao, F. Organic Solar Cells Based on Non-Fullerene Acceptors. Nat. Mater. 2018, 17, 119-128.

4. Inganäs, O. Organic Photovoltaics over Three Decades. Adv. Mater. 2018, 30, 1800388.

5. Menke, S. M.; Holmes, R. J. Exciton Diffusion in Organic Photovoltaic Cells. Energy Environ. Sci. 2014, 7, 499-512.

6. Tamai, Y.; Ohkita, H.; Benten, H.; Ito, S. Exciton Diffusion in Conjugated Polymers: From Fundamental Understanding to Improvement in Photovoltaic Conversion Efficiency. J. Phys. Chem. Lett. 2015, 6, 3417-3428.

7. Mikhnenko, O. V.; Blom, P. W. M.; Nguyen, T.-Q. Exciton Diffusion in Organic Semiconductors. Energy Environ. Sci. 2015, 8, 1867-1888.

8. Hedley, G. J.; Ruseckas, A.; Samuel, I. D. Light Harvesting for Organic Photovoltaics. Chem. Rev. 2017, 117, 796-837.

9. Markov, D. E.; Amsterdam, E.; Blom, P. W. M.; Sieval, A. B.; Hummelen, J. C. Accurate Measurement of the Exciton Diffusion Length in a Conjugated Polymer Using a Heterostructure with a Side-Chain Cross-Linked Fullerene Layer. J. Phys. Chem. A 2005, 109, 5266-5274.

10. Mikhnenko, O. V.; Cordella, F.; Sieval, A. B.; Hummelen, J. C.; Blom, P. W. M.; Loi, M. A. Temperature Dependence of Exciton Diffusion in Conjugated Polymers. J. Phys. Chem. B 2008, $112,11601-11604$. 
11. Mikhnenko, O. V.; Azimi, H.; Scharber, M.; Morana, M.; Blom, P. W. M.; Loi, M. A. Exciton Diffusion Length in Narrow Bandgap Polymers. Energy Environ. Sci. 2012, 5, 6960-6965.

12. Bruno, A.; Reynolds, L. X.; Dyer-Smith, C.; Nelson, J.; Haque, S. A. Determining the Exciton Diffusion Length in a Polyfluorene from Ultrafast Fluorescence Measurements of Polymer/Fullerene Blend Films. J. Phys. Chem. C 2013, 117, 19832-19838.

13. Li, Z.; Lin, J. D. A.; Phan, H.; Sharenko, A.; Proctor, C. M.; Zalar, P.; Chen, Z.; Facchetti, A.; Nguyen, T.-Q. Competitive Absorption and Inefficient Exciton Harvesting: Lessons Learned from Bulk Heterojunction Organic Photovoltaics Utilizing the Polymer Acceptor P(NDI2OD-T2). Adv. Funct. Mater. 2014, 24, 6989-6998.

14. Mikhnenko, O. V.; Kuik, M.; Lin, J.; van der Kaap, N.; Nguyen, T.-Q.; Blom, P. W. TrapLimited Exciton Diffusion in Organic Semiconductors. Adv. Mater. 2014, 26, 1912-1917.

15. Jin, X.-H.; Price, M. B.; Finnegan, J. R.; Boott, C. E.; Richter, J. M.; Rao, A.; Menke, S. M.;

Friend, R. H.; Whittell, G. R.; Manners, I. Long-Range Exciton Transport in Conjugated Polymer Nanofibers Prepared by Seeded Growth. Science 2018, 360, 897-900.

16. Zhang, Y.; Sajjad, M. T.; Blaszczyk, O.; Parnell, A. J.; Ruseckas, A.; Serrano, L. A.; Cooke, G.; Samuel, I. D. W. Large Crystalline Domains and an Enhanced Exciton Diffusion Length Enable Efficient Organic Solar Cells. Chem. Mater. 2019, 31, 6548-6557.

17. Lunt, R. R.; Benziger, J. B.; Forrest, S. R. Relationship between Crystalline Order and Exciton Diffusion Length in Molecular Organic Semiconductors. Adv. Mater. 2010, 22, 1233-1236.

18. Masri, Z.; Ruseckas, A.; Emelianova, E. V.; Wang, L.; Bansal, A. K.; Matheson, A.; Lemke, H. T.; Nielsen, M. M.; Nguyen, H.; Coulembier, O.; Dubois, P.; Beljonne, D.; Samuel, I. D. W. Molecular Weight Dependence of Exciton Diffusion in Poly(3-hexylthiophene). Adv. Energy Mater. 2013, 3, 1445-1453. 
19. Sim, M.; Shin, J.; Shim, C.; Kim, M.; Jo, S. B.; Kim, J.-H.; Cho, K. Dependence of Exciton Diffusion Length on Crystalline Order in Conjugated Polymers. J. Phys. Chem. C 2014, 118, 760766.

20. Chowdhury, M.; Sajjad, M. T.; Savikhin, V.; Hergue, N.; Sutija, K. B.; Oosterhout, S. D.; Toney, M. F.; Dubois, P.; Ruseckas, A.; Samuel, I. D. W. Tuning Crystalline Ordering by Annealing and Additives to Study Its Effect on Exciton Diffusion in a Polyalkylthiophene Copolymer. Phys. Chem. Chem. Phys. 2017, 19, 12441-12451.

21. Tamai, Y.; Matsuura, Y.; Ohkita, H.; Benten, H.; Ito, S. One-Dimensional Singlet Exciton Diffusion in Poly(3-hexylthiophene) Crystalline Domains. J. Phys. Chem. Lett. 2014, 5, 399-403. 22. Ohkita, H.; Tamai, Y.; Benten, H.; Ito, S. Transient Absorption Spectroscopy for Polymer Solar Cells. IEEE J. Sel. Top. Quantum Electron. 2016, 22, 100-111.

23. Pope, M.; Swenberg, C. E. Electronic Processes in Organic Crystals and Polymers. Oxford University Press: Oxford, UK, 1999.

24. Engel, E.; Leo, K.; Hoffmann, M. Ultrafast Relaxation and Exciton-Exciton Annihilation in PTCDA Thin Films at High Excitation Densities. Chem. Phys. 2006, 325, 170-177.

25. Marciniak, H.; Li, X.-Q.; Würthner, F.; Lochbrunner, S. One-Dimensional Exciton Diffusion in Perylene Bisimide Aggregates. J. Phys. Chem. A 2011, 115, 648-654.

26. Baronas, P.; Ščajev, P.; Čerkasovas, V.; Kreiza, G.; Adomėnas, P.; Adomėnienė, O.; Kazlauskas, K.; Adachi, C.; Juršènas, S. Exciton Diffusion in Bifluorene Single Crystals Studied by Light Induced Transient Grating Technique. Appl. Phys. Lett. 2018, 112, 033302.

27. Beljonne, D.; Pourtois, G.; Silva, C.; Hennebicq, E.; Herz, L. M.; Friend, R. H.; Scholes, G. D.; Setayesh, S.; Mullen, K.; Bredas, J. L. Interchain Vs. Intrachain Energy Transfer in AcceptorCapped Conjugated Polymers. PNAS 2002, 99, 10982-10987. 
28. Markov, D. E.; Blom, P. W. M. Anisotropy of Exciton Migration in Poly(p-phenylene vinylene). Phys. Rev. B 2006, 74, 085206.

29. Tapping, P. C.; Clafton, S. N.; Schwarz, K. N.; Kee, T. W.; Huang, D. M. Molecular-Level Details of Morphology-Dependent Exciton Migration in Poly(3-hexylthiophene) Nanostructures. J. Phys. Chem. C 2015, 119, 7047-7059.

30. Kawashima, K.; Osaka, I.; Takimiya, K. Effect of Chalcogen Atom on the Properties of Naphthobischalcogenadiazole-Based $\pi$-Conjugated Polymers. Chem. Mater. 2015, 27, 6558-6570. 31. Kawashima, K.; Tamai, Y.; Ohkita, H.; Osaka, I.; Takimiya, K. High-Efficiency Polymer Solar Cells with Small Photon Energy Loss. Nat. Commun. 2015, 6, 10085.

32. Spano, F. C. Modeling Disorder in Polymer Aggregates: The Optical Spectroscopy of Regioregular Poly(3-hexylthiophene) Thin Films. J Chem Phys 2005, 122, 234701.

33. Spano, F. C. Absorption in Regio-Regular Poly(3-hexyl)Thiophene Thin Films: Fermi Resonances, Interband Coupling and Disorder. Chem. Phys. 2006, 325, 22-35.

34. Clark, J.; Chang, J.-F.; Spano, F. C.; Friend, R. H.; Silva, C. Determining Exciton Bandwidth and Film Microstructure in Polythiophene Films Using Linear Absorption Spectroscopy. Appl. Phys. Lett. 2009, 94, 163306.

35. Erb, T.; Zhokhavets, U.; Gobsch, G.; Raleva, S.; Stuhn, B.; Schilinsky, P.; Waldauf, C.; Brabec, C. J. Correlation between Structural and Optical Properties of Composite Polymer/Fullerene Films for Organic Solar Cells. Adv. Funct. Mater. 2005, 15, 1193-1196.

36. Agbolaghi, S.; Zenoozi, S. A Comprehensive Review on Poly(3-alkylthiophene)-Based Crystalline Structures, Protocols and Electronic Applications. Org. Electron. 2017, 51, 362-403. 
37. Clark, J.; Silva, C.; Friend, R. H.; Spano, F. C. Role of Intermolecular Coupling in the Photophysics of Disordered Organic Semiconductors: Aggregate Emission in Regioregular Polythiophene. Phys. Rev. Lett. 2007, 98, 206406.

38. Spano, F. C.; Clark, J.; Silva, C.; Friend, R. H. Determining Exciton Coherence from the Photoluminescence Spectral Line Shape in Poly(3-hexylthiophene) Thin Films. J. Chem. Phys. 2009, 130, 074904.

39. Yamagata, H.; Spano, F. C. Interplay between Intrachain and Interchain Interactions in Semiconducting Polymer Assemblies: The HJ-Aggregate Model. J. Chem. Phys. 2012, 136, 184901.

40. Spano, F. C.; Silva, C. H- and J-Aggregate Behavior in Polymeric Semiconductors. Annu. Rev. Phys. Chem. 2014, 65, 477-500.

41. Niles, E. T.; Roehling, J. D.; Yamagata, H.; Wise, A. J.; Spano, F. C.; Moulé, A. J.; Grey, J. K. J-Aggregate Behavior in Poly-3-hexylthiophene Nanofibers. J. Phys. Chem. Lett. 2012, 3, 259263.

42. Cook, S.; Han, L. Y.; Furube, A.; Katoh, R. Singlet Annihilation in Films of Regioregular Poly(3-hexylthiophene): Estimates for Singlet Diffusion Lengths and the Correlation between Singlet Annihilation Rates and Spectral Relaxation. J. Phys. Chem. C 2010, 114, 10962-10968.

43. Lin, J. D. A.; Mikhnenko, O. V.; Chen, J.; Masri, Z.; Ruseckas, A.; Mikhailovsky, A.; Raab, R. P.; Liu, J.; Blom, P. W. M.; Loi, M. A.; García-Cervera, C. J.; Samuel, I. D. W.; Nguyen, T.-Q. Systematic Study of Exciton Diffusion Length in Organic Semiconductors by Six Experimental Methods. Mater. Horiz. 2014, 1, 280-285. 
44. Rao, A.; Chow, P. C.; Gélinas, S.; Schlenker, C. W.; Li, C. Z.; Yip, H. L.; Jen, A. K.; Ginger,

D. S.; Friend, R. H. The Role of Spin in the Kinetic Control of Recombination in Organic Photovoltaics. Nature 2013, 500, 435-439.

45. Gélinas, S.; Rao, A.; Kumar, A.; Smith, S. L.; Chin, A. W.; Clark, J.; van der Poll, T. S.; Bazan,

G. C.; Friend, R. H. Ultrafast Long-Range Charge Separation in Organic Semiconductor Photovoltaic Diodes. Science 2014, 343, 512-516.

46. Chow, P. C.; Gélinas, S.; Rao, A.; Friend, R. H. Quantitative Bimolecular Recombination in Organic Photovoltaics through Triplet Exciton Formation. J. Am. Chem. Soc. 2014, 136, 34243429.

47. Tamai, Y.; Fan, Y.; Kim, V. O.; Ziabrev, K.; Rao, A.; Barlow, S.; Marder, S. R.; Friend, R. H.; Menke, S. M. Ultrafast Long-Range Charge Separation in Nonfullerene Organic Solar Cells. ACS Nano 2017, 11, 12473-12481. 